\title{
Evaluation of Central Corneal Thickness Using Corneal Dynamic Scheimpflug Analyzer Corvis ST and Comparison with Pentacam Rotating Scheimpflug System and Ultrasound Pachymetry in Normal Eyes
}

\author{
Ayong Yu, ${ }^{1,2}$ Weiqi Zhao, ${ }^{1,2}$ Giacomo Savini, ${ }^{3}$ Zixu Huang, ${ }^{1}$ Fangjun Bao, ${ }^{1,2}$ \\ Weicong Lu, ${ }^{1,2}$ Qinmei Wang, ${ }^{1,2}$ and Jinhai Huang ${ }^{1,2}$ \\ ${ }^{1}$ School of Ophthalmology and Optometry, Wenzhou Medical University, 270 West Xueyuan Road, Wenzhou, Zhejiang 325027, China \\ ${ }^{2}$ Key Laboratory of Vision Science, Ministry of Health of the People's Republic of China, Wenzhou, Zhejiang, China \\ ${ }^{3}$ G. B. Bietti Foundation IRCCS, Rome, Italy
}

Correspondence should be addressed to Qinmei Wang; wqm6@mail.eye.ac.cn and Jinhai Huang; vip999vip@163.com

Received 7 October 2015; Accepted 16 November 2015

Academic Editor: David P. Piñero

Copyright (C) 2015 Ayong Yu et al. This is an open access article distributed under the Creative Commons Attribution License, which permits unrestricted use, distribution, and reproduction in any medium, provided the original work is properly cited.

Purpose. To assess the repeatability and reproducibility of central corneal thickness (CCT) measurements by corneal dynamic Scheimpflug analyzer Corvis ST in normal eyes and compare the agreement with Pentacam rotating Scheimpflug System and ultrasound pachymetry. Methods. 84 right eyes underwent Corvis ST measurements performed by two operators. The test-retest repeatability (TRT), within-subject coefficient of variation $(\mathrm{CoV})$, and intraclass correlation coefficient (ICC) were used to evaluate the intraoperator repeatability and interoperator reproducibility. CCT measurements also were obtained from Pentacam and ultrasound pachymetry by the first operator. The agreement between the three devices was evaluated with $95 \%$ limits of agreement (LoA) and Bland-Altman plots. Results. Corvis ST showed high repeatability as indicated by TRT $\leq 13.0 \mu \mathrm{m}, \mathrm{CoV}<0.9 \%$, and ICC $>0.97$. The interoperator reproducibility was also excellent. The CoV was $<0.9 \%$, and ICC was $>0.97$. Corvis ST showed significantly lower values than Pentacam and ultrasound pachymetry $(P<0.001)$. The $95 \%$ LoA between Corvis ST and Pentacam or ultrasound pachymetry were -15.8 to $9.5 \mu \mathrm{m}$ and -27.9 to $12.3 \mu \mathrm{m}$, respectively. Conclusions. Corvis ST showed excellent repeatability and interoperator reproducibility of CCT measurements in normal eyes. Corvis ST is interchangeable with Pentacam but not with ultrasound pachymetry.

\section{Introduction}

Accurate assessment of the central corneal thickness (CCT) has become extremely important in ophthalmologic examinations. Preoperatively, it helps the ophthalmologist to safely plan corneal refractive procedures and screen for refractive surgery candidates, in order to reduce the risk of postoperative complications [1]. Besides, CCT measurements play a crucial role in the diagnosis and management of glaucoma because the value of intraocular pressure should be adjusted in accordance with CCT [2]. CCT measurements also play an important role in the diagnosis of corneal diseases, such as Fuchs' corneal dystrophy and keratoconus [3, 4].
For many years, ultrasound pachymetry has been the most frequently used method to measure CCT because it is relatively inexpensive and easy to use and has high intraoperator repeatability $[2,5]$. Nevertheless, ultrasound pachymetry has certain disadvantages, such as corneal-probe contact, the need for topical anesthesia, and the risk for transmission of infections and corneal epithelial lesions [6]. Besides, the reliability of ultrasound pachymetry results depends on the operator's skill when placing the probe perpendicularly to the cornea. Over the last decade, many noncontact devices have been developed. Among these, Scheimpflug technology plays a major role, including Pentacam (Oculus, Wetzlar, Germany), Sirius (Costruzione Strumenti Oftalmici, 
Florence, Italy), Galilei (Ziemer, Port, Switzerland), and TMS-5 (Tomey, Nagoya, Japan). Previous studies have shown that common used device Pentacam has high intraoperator repeatability and interoperator reproducibility for CCT measurements [7-9].

The corneal dynamic Scheimpflug analyzer Corvis ST (Oculus Optikgeräte, Inc., Wetzlar, Germany) is relatively new, noncontact corneal biomechanics equipment, which is composed of an air puff indentation system and ultrahigh-speed Scheimpflug technology. The ultra-high-speed Scheimpflug camera has a blue light LED and acquires the deformation process at 4330 frames/s with an $8 \mathrm{~mm}$ horizontal coverage. Because of the air impulse, the cornea experiences three stages: first applanation, highest concavity, and second applanation. Furthermore, CCT is obtained by the corneal initial state of the central horizontal cross-section diagram through the Scheimpflug technology. Few studies [10-12] have evaluated the intraoperator repeatability of CCT measurements obtained by this device in normal population. Ali et al. [10] evaluated the intersession reproducibility of the CCT at different times of the day with this device. Chen et al. [13] assessed the intraoperator repeatability and interobserver reproducibility in virgin and post-PRK eyes but only applied single value to evaluate interobserver reproducibility. However, to our knowledge, the interoperator reproducibility of Corvis ST for CCT measurements using both single and average measurement value methods in normal eyes has not yet been evaluated.

The purpose of the present study was to prospectively assess the intraoperator repeatability and interoperator reproducibility of CCT measurements using both single and average methods acquired from the Corvis ST in normal eyes and compare the agreement with Pentacam and ultrasound pachymetry.

\section{Subjects and Methods}

This prospective study was conducted on normal subjects recruited from the staff and students of the Eye Hospital of Wenzhou Medical University. The research protocol conformed to the tenets of the Declaration of Helsinki and was approved by the Office of Research Ethics, Eye Hospital of Wenzhou Medical University. Informed consent was acquired from all subjects after explaining the purpose of the study.

The exclusion criteria were active ocular pathology, any history of ocular surgery or trauma, recent contact lens wear (soft contact lens within two weeks and rigid contact lens within four weeks), systemic diseases with eye symptoms, and intraocular pressure $>21 \mathrm{mmHg}$.

All subjects underwent a comprehensive ophthalmic examination, including uncorrected distance visual acuity, best-corrected visual acuity, slit-lamp microscopy, noncontact tonometer, and fundus examinations. Subsequently, we applied Pentacam, Corvis ST, and ultrasound pachymetry to measure CCT. To avoid any effect of the ultrasound probe and topical anesthetic on the cornea, the two noncontact pieces of equipment were used first. The sequence of measurements with Pentacam and Corvis ST was randomly chosen. Measurements were acquired from the right eyes of subjects to avoid structural similarities between fellow eyes [14]. In order to minimize the diurnal variation on CCT readings, all measurements were performed from 10:00 to 17:00. The subjects were required to completely blink twice before measurements, in order to form an optically smooth tear film on the cornea.

Corvis ST examination applies four red alignment marks to position the center of the cornea on the computer screen. Once positioned successfully, a puff of air with a pressure of $25 \mathrm{kPa}$ is emitted automatically from the instrument aimed at the cornea at a distance of $11 \mathrm{~mm}$. During the examination, the Scheimpflug camera records corneal deformation process and CCT. The process was repeated until three acceptable readings were obtained. The three CCT measurements obtained by each examiner were used to evaluate the intraoperator repeatability. The mean value of three successive measurements and the first measurement by each examiner were used to analyze the interoperator reproducibility.

The Pentacam was used as previously described $[8,9]$. Briefly, the subject was instructed to sit, open both eyes, and fixate on a target within the device. The real-time image of the subject's eye on the computer screen was adjusted according to the pupil edge, center, and the corneal apex by moving the joystick. To avoid operator-dependent variables, the automatic release mode was applied. The Pentacam would automatically measure when correct alignment with the corneal apex and focus was achieved. Only when the "examination quality specification" reading showed $\mathrm{OK}$, it was recorded; otherwise it was excluded and remeasured until three valid readings were obtained.

After the CCT measurements were obtained by Pentacam and Corvis ST, an A-scan ultrasound pachymetry (SP-3000, Tomey Inc., Nagoya, Japan) was used. Before the measurements, the instrument was calibrated by an experienced technician. First, the cornea was anesthetized with topical $0.5 \%$ proparacaine hydrochloride (Alcaine; Alcon Laboratories, TX, USA). Then, the subject in the supine position was asked to fixate on a target on the ceiling. The examiner placed the pachymeter probe on the central cornea as perpendicularly as possible. Then, five consecutive measurements were obtained, of which the highest and lowest were excluded, and the remaining three were recorded.

Statistical Analysis. Statistical analysis was performed using SPSS software for Windows version 21 (IBM Corporation, USA) and Microsoft Office Excel (Microsoft Corp., WA, USA). $P<0.05$ was considered to be statistically significant. The distributions were checked by Kolmogorov-Smirnov test, which showed that the data were normally distributed $(P>$ $0.05)$. Results were presented as means \pm standard deviations.

To assess the intraoperator repeatability of Corvis ST, within-subject standard deviation $\left(S_{w}\right)$, test-retest repeatability (TRT), within-subject coefficient of variation (CoV), and intraclass correlation coefficient (ICC) were calculated for the three successive measurements obtained by the two operators. The TRT is 2.77 times $S_{w}$, which represents an interval within which $95 \%$ of the differences between 
TABLE 1: Intraobserver repeatability of the corneal dynamic Scheimpflug analyzer Corvis ST in measuring central corneal thickness $(\mathrm{N}=84)$.

\begin{tabular}{lccccc}
\hline Operator & Mean $(\mu \mathrm{m}) \pm \mathrm{SD}$ & $S_{w}(\mu \mathrm{m})$ & TRT $(\mu \mathrm{m})$ & CoV $(\%)$ & ICC $(95 \% \mathrm{CI})$ \\
\hline 1st & $535.9 \pm 27.0$ & 4.8 & 13.0 & 0.87 & 0.971 \\
2nd & $537.4 \pm 27.6$ & 4.7 & 13.0 & 0.87 & 0.958 to 0.980$)$ \\
& & & & 0.972 \\
\hline
\end{tabular}

$\mathrm{SD}=$ standard deviation, $S_{w}=$ within-subject standard deviation, TRT = test-retest repeatability $\left(2.77 S_{w}\right), \mathrm{CoV}=$ within-subject coefficient of variation, and ICC $=$ intraclass correlation coefficient.

TABLE 2: Interobserver reproducibility of central corneal thickness readings using average (from average of 3 consecutive readings from each observer) and single (from the first reading from each observer) measurement by the corneal dynamic Scheimpflug analyzer Corvis ST.

\begin{tabular}{lccccc}
\hline Parameter & Mean $(\mu \mathrm{m}) \pm \mathrm{SD}$ & $S_{w}(\mu \mathrm{m})$ & TRT $(\mu \mathrm{m})$ & CoV $(\%)$ & ICC $(95 \% \mathrm{CI})$ \\
\hline Average & $536.6 \pm 27.2$ & 3.5 & 9.7 & 0.65 & 0.984 \\
Single & $536.4 \pm 27.5$ & 4.5 & 12.6 & 0.85 & 0.973 to 0.990$)$ \\
\hline
\end{tabular}

$\mathrm{SD}=$ standard deviation, $S_{w}=$ within-subject standard deviation, TRT $=$ test-retest repeatability $\left(2.77 S_{w}\right), \mathrm{CoV}=$ within-subject coefficient of variation, and ICC $=$ intraclass correlation coefficient.

TABLE 3: Comparison of the central corneal thickness readings obtained using the corneal dynamic Scheimpflug analyzer Corvis ST, Pentacam rotating Scheimpflug system, and ultrasound pachymetry.

\begin{tabular}{lccc}
\hline Device pairings & Mean difference $(\mu \mathrm{m}) \pm \mathrm{SD}$ & $95 \% \operatorname{LoA}(\mu \mathrm{m})$ & $P$ value \\
\hline Corvis-Pentacam & $-3.2 \pm 6.5$ & -15.8 to 9.5 & $<0.001$ \\
Corvis-USP & $-7.8 \pm 10.3$ & -27.9 to 12.3 & $<0.001$ \\
\hline
\end{tabular}

$\mathrm{USP}=$ ultrasound pachymetry, $\mathrm{SD}=$ standard deviation.

measurements are expected to lie [15]. The CoV is defined as the ratio of $S_{w}$ to the overall mean. A lower $\mathrm{CoV}$ is closely related to higher repeatability. The ICC represents the consistency of measurement. The closer the ICC is to 1 , the better the consistency of measurement is. To evaluate the interoperator reproducibility of Corvis ST, the average method (the difference between the mean of the three successive measurements obtained by the two operators) and the single method (the first measurement of each operator) were used. Then, the interoperator $S_{w}$, TRT, CoV, and ICC were also calculated.

For multiple comparisons between CCT measurements obtained by Corvis ST and Pentacam or ultrasound pachymetry, the repeated-measures analysis of variance (ANOVA) with Bonferroni post hoc comparison test was used. Furthermore, the $95 \%$ limits of agreement (LoA) were calculated and Bland-Altman plots were produced to evaluate the agreement on the CCT measurements between Corvis ST versus Pentacam and Corvis ST versus ultrasound pachymetry [16]. The 95\% LoA are defined as mean \pm 1.96 SD, which represent an interval within which $95 \%$ of the differences between readings are expected to lie [8].

\section{Results}

This study enrolled 84 right eyes of 84 subjects ( 38 males and 46 females). The mean age of the subjects was $27.30 \pm 6.06$ years (range 18 to 49 years). The mean spherical equivalent refraction was $-4.12 \pm 2.66 \mathrm{D}$ (range -10.50 to $+0.50 \mathrm{D})$.

3.1. Intraoperator Repeatability. The CCT measurements obtained using Corvis ST showed excellent intraoperator repeatability for both operators (Table 1 ). The TRT values were $\leq 13 \mu \mathrm{m}$, the $\mathrm{CoV}$ values were $<0.9 \%$, and the ICC values were $>0.97$.

3.2. Interoperator Reproducibility. The mean $\pm \mathrm{SD}$ of CCT, $S_{w}$, TRT, CoV, and ICC of Corvis ST are shown in Table 2, which demonstrate high interoperator reproducibility. In the average method, the TRT was $9.7 \mu \mathrm{m}$, the $\mathrm{CoV}$ was $0.65 \%$, and the ICC was 0.98. In the single method, the TRT was $12.6 \mu \mathrm{m}$, the $\mathrm{CoV}$ was $0.85 \%$, and the ICC was 0.97 . Obviously, the error of the average method was smaller than the single method.

3.3. Agreement between Corvis ST, Pentacam, and Ultrasound Pachymetry. The mean CCT readings using Corvis ST, Pentacam, and ultrasound pachymetry were $535.9 \pm$ $27.0 \mu \mathrm{m}, 539.0 \pm 25.70 \mu \mathrm{m}$, and $543.7 \pm 27.52 \mu \mathrm{m}$, respectively. The CCT readings measured by Corvis ST were significantly thinner than Pentacam $(P<0.001)$. Bland-Altman analysis confirmed these results (Table 3 and Figure 1$)$. The mean difference was $-3.2 \mu \mathrm{m}$ (95\% LoA, -15.8 to $9.5 \mu \mathrm{m})$. 


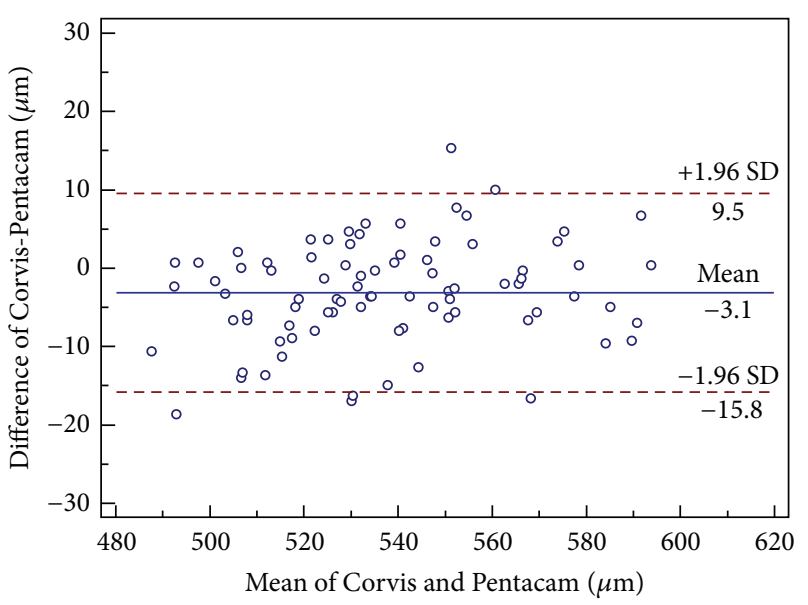

Figure 1: Bland-Altman plot demonstrating central corneal thickness measurements obtained using corneal dynamic Scheimpflug analyzer Corvis ST and Pentacam rotating Scheimpflug system against the mean values for both devices. The $95 \%$ limits of agreement are represented as the upper and lower lines.

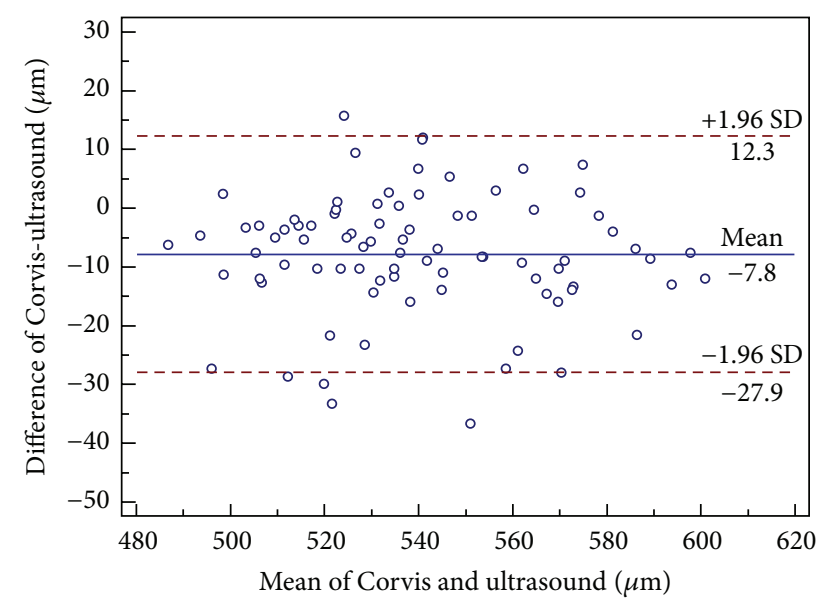

FIGURE 2: Bland-Altman plot demonstrating central corneal thickness measurements obtained using corneal dynamic Scheimpflug analyzer Corvis ST and ultrasound pachymetry against the mean values for both devices. The $95 \%$ limits of agreement are represented as the upper and lower lines.

CCT measurements between Corvis ST and ultrasound pachymetry were significantly different $(P<0.001)$. The mean difference was $-7.8 \mu \mathrm{m}$ (95\% LoA, -27.9 to $12.3 \mu \mathrm{m})$ (Table 3 and Figure 2).

\section{Discussion}

The present study was prospectively designed to assess (1) the intraoperator repeatability and (2) the interoperator reproducibility by applying the single and average methods on CCT measurements obtained from Corvis ST in normal eyes and (3) to evaluate the agreement between Corvis ST, Pentacam, and ultrasound pachymetry. The TRT was $\leq 13.0 \mu \mathrm{m}$, the $\mathrm{CoV}$ was $<0.90 \%$, and the ICC was $>0.97$, which represented high intraoperator repeatability in CCT readings using Corvis ST in normal eyes. Ali et al. [10] obtained similar results in normal eyes, with TRT, CoV, and ICC of $27 \mu \mathrm{m}, 1.83 \%$, and 0.95 , respectively. Hon and Lam [11] reported TRT, CoV, and ICC of $15.34 \mu \mathrm{m}, 1.01 \%$, and 0.96 , respectively, in normal subjects. Nemeth et al. [12] obtained similar ICC of 0.97 and CoV of $0.8 \%$ for CCT in normal eyes. Salvetat et al. [17] reported ICC of 0.99 in normal subjects and primary open-angle glaucoma patients. Chen et al. [13] obtained similar results in virgin eyes, with TRT, CoV, and ICC of $12.56 \mu \mathrm{m}, 0.69 \%$, and 0.99 , respectively. However, they reported worse results in post-PRK eyes, with TRT and CoV of $22.61 \mu \mathrm{m}$ and $2.29 \%$, respectively.

Previous studies had assessed the intraoperator repeatability of CCT values using other Scheimpflug systems and specular microscopes, such as Pentacam, Sirius, Galilei, Orbscan II (Bausch \& Lomb, Rochester, NY, USA), and SP02 (Costruzione Strumenti Oftalmici, Italy). Huang et al. [7] evaluated the intraoperator repeatability of Sirius and Pentacam, a rotating Scheimpflug camera combined with a Placido disk corneal topographer and a rotating Scheimpflug camera, respectively, in normal subjects. They indicated that the TRT values were $8.79 \mu \mathrm{m}$ and $9.65 \mu \mathrm{m}$, the $\mathrm{CoV}$ values were $0.59 \%$ and $0.65 \%$, and the ICC values were 0.98 and 0.98 , respectively. These results were slightly better than our results with Corvis ST. Al-Mohtaseb et al. [18] assessed the intraoperator repeatability of the Galilei, a dual rotating Scheimpflug camera combined with a Placido disk, in normal eyes. The CoV was $0.36 \%$ and the ICC was 0.99 , which were also slightly better than our results with Corvis ST. Maldonado et al. [19] studied the intraoperator repeatability of the Orbscan II, a scanning-slit combined with Placido disc topography, with TRT and CoV of $20.2 \mu \mathrm{m}$ and $1.5 \%$, respectively, which were worse than our results. Bao et al. [20] assessed the intraoperator repeatability of SP-02, a noncontact specular microscope, in normal eyes with TRT, CoV, and ICC of $18.67 \mu \mathrm{m}, 1.23 \%$, and 0.97 , respectively, which were worse than our results with Corvis ST. These indirect comparisons indicate that the above-mentioned devices have high intraoperator repeatability, with Galilei being the best. The Galilei has two opposite Scheimpflug cameras and can calculate the average value of the CCT obtained by the two cameras. This reduces the artifact error caused by ocular movements and increases repeatability [21]. Therefore, future studies should compare Corvis ST, Galilei, and Sirius.

In the present study, we analyzed the interoperator reproducibility of CCT measurements acquired with Corvis ST by applying the single and average methods. The TRT and CoV of the mean values were smaller than the single values. Chen and Lam [22, 23] demonstrated that the width of 95\% LoA was reduced by using an averaged result rather than the first result of each visit. Ali et al. [10] assessed the reproducibility of the CCT with the device in normal eyes, which was intersession reproducibility at different times of the day. In their study, TRT, CoV, and ICC were $11.0 \mu \mathrm{m}, 7.41 \%$, and 0.995 , respectively, while they were $9.70 \mu \mathrm{m}, 0.65 \%$, and 0.984 in the current study, respectively. Our results were obviously better than their results because our results were accomplished within 30 minutes, which eliminated the effects of different 
TABLE 4: Summary of previous studies for the agreement of CCT measurements obtained by other Scheimpflug systems, Orbscan II and specular microscopes in comparison to Pentacam or ultrasound pachymetry.

\begin{tabular}{|c|c|c|c|c|c|}
\hline Author (year) & Patients/eyes & Device pairings & Mean difference $(\mu \mathrm{m}) \pm \mathrm{SD}$ & $P$ value & 95\% LoA $(\mu \mathrm{m})$ \\
\hline \multirow{3}{*}{ Lanza et al. [27] (2015) } & \multirow{3}{*}{$102 / 102$} & Pentacam-Orbscan II & $13.66 \pm 16.53$ & $<0.0001$ & -18.74 to 46.06 \\
\hline & & Sirius-Orbscan II & $15.18 \pm 17.16$ & $<0.0001$ & -18.45 to 48.81 \\
\hline & & Sirius-Pentacam & $1.52 \pm 6.21$ & 0.015 & -10.65 to 13.69 \\
\hline \multirow{2}{*}{ Khaja et al. [28] (2015) } & \multirow{2}{*}{$32 / 32$} & USP-Orbscan II & $2.8 \pm 0.28$ & NA & -30.15 to 24.40 \\
\hline & & USP-specular microscopy & $8.69 \pm 1.24$ & NA & -8.82 to 27.4 \\
\hline \multirow{2}{*}{ Smedowski et al. [29] (2014) } & \multirow{2}{*}{$76 / 152$} & Corvis ST-Pentacam & NA & $>0.05$ & NA \\
\hline & & Corvis ST-USP & NA & $>0.05$ & NA \\
\hline \multirow{3}{*}{ Huang et al. [30] (2014) } & \multirow{3}{*}{$66 / 66$} & Pentacam-Sirius & $-3.3 \pm 5.2$ & $<0.001$ & -13.6 to 6.9 \\
\hline & & Pentacam-Galilei & $-9.3 \pm 3.7$ & $<0.001$ & -16.6 to -2.0 \\
\hline & & Sirius-Galilei & $-6.0 \pm 4.0$ & $<0.001$ & -13.8 to 1.9 \\
\hline \multirow{3}{*}{ Anayol et al. [31] (2014) } & \multirow{3}{*}{$32 / 32$} & Galilei-Pentacam & $13.93 \pm 0.88$ & $<0.001$ & 11.74 to 16.12 \\
\hline & & Galilei-Sirius & $14.66 \pm 0.69$ & $<0.001$ & 12.96 to 16.37 \\
\hline & & Pentacam-Sirius & $0.73 \pm 0.93$ & 1.0 & -1.50 to 3.02 \\
\hline Maresca et al. [32] (2014) & $35 / 35$ & Sirius-USP & $-13.9 \pm 14.4$ & $<0.001$ & -42.2 to 14.4 \\
\hline \multirow{3}{*}{ Feizi et al. [33] (2014) } & \multirow{3}{*}{$88 / 88$} & USP-Orbscan & $-14.5 \pm 22.9$ & $<0.001$ & -59.4 to 30.4 \\
\hline & & USP-Galilei & $-16.0 \pm 19.6$ & $<0.001$ & -54.5 to 22.5 \\
\hline & & Orbscan-Galilei & $-1.5 \pm 17.0$ & 0.99 & -34.8 to 31.9 \\
\hline De La Parra-Colín et al. [34] (2014) & $16 / 16$ & Sirius-Pentacam & $-10.1 \pm 9.0$ & NA & -27.7 to 7.5 \\
\hline Jorgel et al. [35] (2013) & $50 / 50$ & Sirius-USP & $4.68 \pm 10.47$ & 0.003 & -15.84 to 25.20 \\
\hline Bayhan et al. [36] (2014) & $50 / 50$ & USP-Sirius & $17.58 \pm 8.13$ & $<0.001$ & 15.27 to 19.89 \\
\hline Huang et al. [37] (2013) & $43 / 43$ & Sirius-USP & $6.88 \pm 6.77$ & 0.000 & -6.39 to 20.14 \\
\hline \multirow{2}{*}{ Nassiri et al. [38] (2014) } & \multirow{2}{*}{$32 / 61$} & Pentacam-USP & $-1 \pm 9$ & 0.32 & -20 to 17 \\
\hline & & Orbscan II-USP & $6 \pm 14$ & $<0.001$ & -21 to 33 \\
\hline Al Farhan et al. [39] (2013) & $30 / 30$ & USP-specular microscopy & $-2.40 \pm 9.10$ & 0.16 & -38.70 to 39.90 \\
\hline \multirow{3}{*}{ Tai et al. [40] (2013) } & \multirow{3}{*}{$92 / 184$} & Pentacam-USP & $10.08 \pm 10.96$ & 0.012 & -11.40 to 31.56 \\
\hline & & Specular microscopy-USP & $-20.49 \pm 8.91$ & $<0.001$ & -37.95 to -3.04 \\
\hline & & $\begin{array}{l}\text { Specular } \\
\text { microscopy-Pentacam }\end{array}$ & $-30.57 \pm 10.26$ & $<0.001$ & -59.69 to -10.45 \\
\hline Chen et al. [41] (2012) & $35 / 35$ & Pentacam-USP & $5.27 \pm 9.55$ & 0.007 & -24.0 to -13.4 \\
\hline Aramberri et al. [42] (2012) & $35 / 35$ & Pentacam-Galilei & $-2.76 \pm 4.52$ & $<0.01$ & -6.1 to 11.6 \\
\hline \multirow{8}{*}{ González-Pérez et al. [43] (2011) } & \multirow{8}{*}{$22 / 22$} & USP-Pentacam & $3 \pm 10$ & 0.233 & -16.2 to 21.2 \\
\hline & & USP-Orbscan II & $32 \pm 15$ & $<0.001$ & 3.1 to 60.8 \\
\hline & & USP-specular microscopy & $26 \pm 37$ & 0.004 & -46.2 to 97.8 \\
\hline & & Pentacam-Orbscan II & $29 \pm 11$ & $<0.001$ & 7.2 to 51.6 \\
\hline & & $\begin{array}{l}\text { Pentacam-specular } \\
\text { microscopy }\end{array}$ & $23 \pm 32$ & 0.003 & -40.2 to 86.2 \\
\hline & & $\begin{array}{l}\text { Orbscan II-specular } \\
\text { microscopy }\end{array}$ & $-6 \pm 35$ & 0.399 & -74.8 to 62.0 \\
\hline & & USP-Orbscan II & $-15 \pm 17$ & 0.001 & -47.9 to 18.7 \\
\hline & & Pentacam-Orbscan II & $-17 \pm 14$ & $<0.001$ & -43.9 to 9.8 \\
\hline
\end{tabular}

times on the CCT. Chen et al. [13] measured the interoperator reproducibility of the CCT by the single method in virgin and post-PRK eyes, and the TRT, CoV, and ICC were $13.24 \mu \mathrm{m}$, $0.72 \%$, and 0.98 in virgin eyes and $9.89 \mu \mathrm{m}, 0.58 \%$, and 1.00 in post-PRK eyes, respectively, which were similar to our results using the single method. Salvetat et al. [17] only applied ICC to assess the interoperator reproducibility, which was 0.99 in normal subjects and primary open-angle glaucoma patients. We believe that ours is the first study to evaluate the interoperator reproducibility of Corvis ST using the average and single methods with TRT, CoV, and ICC.

In addition, we compared the CCT readings between Corvis ST, Pentacam, and ultrasound pachymetry in normal eyes. Corvis ST had a slightly lower CCT measurement as compared to Pentacam with a mean of $3.2 \mu \mathrm{m}$. Meanwhile, Corvis ST significantly underestimated CCT as compared 
to ultrasound pachymetry with an average of $7.8 \mu \mathrm{m}$. The 95\% LoA between Corvis ST and Pentacam were narrow and comparable, with the CCT diurnal pachymetric variation range of -11 to $11 \mu \mathrm{m}$ [24]. Therefore, Corvis ST and Pentacam could be interchangeably used in normal eyes in most clinical applications. However, Corvis ST cannot be interchangeably used with ultrasound pachymetry in normal eyes because of broad 95\% LoA between the two devices. Our results were similar to or better than those previously reported when investigating agreement of CCT measurements obtained from other Scheimpflug systems, Orbscan II, and specular microscopes with respect to Pentacam or ultrasound pachymetry (Table 4). Several reasons may explain the difference in CCT readings between Corvis ST and ultrasound pachymetry. Firstly, topical $0.5 \%$ proparacaine hydrochloride may cause corneal thickness to increase by $8.6 \mu \mathrm{m}$ in 80 seconds [25]. Secondly, the accuracy of ultrasound pachymetry depends on the operator's proficiency and whether the corneal probe is perpendicularly placed on the center of the cornea. Thirdly, if the posterior surface reflection point is closer to the anterior chamber, the CCT measurement is thicker than the actual value [26].

The present study had some limitations. First, we only assessed the intraoperator repeatability and interoperator reproducibility in normal subjects and did not include keratoconus, glaucoma, or postrefractive surgery patients. Further research is needed to assess the intraoperator repeatability and interoperator reproducibility in the above-mentioned patients. Second, our study is restricted by the different algorithms each device uses for obtaining the CCT. The CCT obtained by Corvis ST and Pentacam are derived from the corneal apex. However, ultrasound pachymetry is performed over the pupil center, and its position depends on the operator's experience.

In conclusion, Corvis ST showed high intraoperator repeatability and interoperator reproducibility of CCT measurements in normal eyes. Corvis ST and Pentacam showed excellent agreement, which suggests that the two devices may be interchangeably used for CCT measurements in the clinical setting. However, the CCT readings between Corvis ST and ultrasound pachymetry are not directly interchangeable owing to the relatively wide $95 \%$ LoA.

\section{Conflict of Interests}

The authors have no proprietary or commercial interest in any materials discussed in this paper.

\section{Authors' Contribution}

Ayong Yu and Weiqi Zhao have contributed equally as first authors.

\section{Acknowledgments}

This work is supported in part by the National Natural Science Foundation of China (81300807); Foundation of Wenzhou City Science \& Technology Bureau (J20140014 and Y20140619); Health Bureau of Zhejiang Province (2016RCB013); Zhejiang Provincial \& Ministry of Health Research Fund for Medical Sciences (WKJ-ZJ-1530); National Science and Technology Major Project (2014ZX09303301). The contribution of G. B. Bietti Foundation IRCCS was supported by the Italian Ministry of Health and Fondazione Roma.

\section{References}

[1] Z. Wang, J. Chen, and B. Yang, "Posterior corneal surface topographic changes after laser in situ keratomileusis are related to residual corneal bed thickness," Ophthalmology, vol. 106, no. 2, pp. 406-410, 1999.

[2] M. J. Doughty and M. L. Zaman, "Human corneal thickness and its impact on intraocular pressure measures: a review and metaanalysis approach," Survey of Ophthalmology, vol. 44, no. 5, pp. 367-408, 2000.

[3] G. U. Auffarth, L. Wang, and H. E. Völcker, "Keratoconus evaluation using the Orbscan Topography System," Journal of Cataract and Refractive Surgery, vol. 26, no. 2, pp. 222-228, 2000.

[4] Ö. Ö. Uçakhan, M. Özkan, and A. Kanpolat, "Corneal thickness measurements in normal and keratoconic eyes: pentacam comprehensive eye scanner versus noncontact specular microscopy and ultrasound pachymetry," Journal of Cataract and Refractive Surgery, vol. 32, no. 6, pp. 970-977, 2006.

[5] H. A. Swarbrick, G. Wong, and D. J. O'Leary, "Corneal response to orthokeratology," Optometry and Vision Science, vol. 75, no. 11, pp. 791-799, 1998.

[6] Y. Barkana, Y. Gerber, U. Elbaz et al., "Central corneal thickness measurement with the Pentacam Scheimpflug system, optical low-coherence reflectometry pachymeter, and ultrasound pachymetry," Journal of Cataract and Refractive Surgery, vol. 31, no. 9, pp. 1729-1735, 2005.

[7] J. Huang, X. Ding, G. Savini et al., "A comparison between scheimpflug imaging and optical coherence tomography in measuring corneal thickness," Ophthalmology, vol. 120, no. 10, pp. 1951-1958, 2013.

[8] J. Huang, K. Pesudovs, A. Yu et al., "A comprehensive comparison of central corneal thickness measurement," Optometry and Vision Science, vol. 88, no. 8, pp. 940-949, 2011.

[9] R. Khoramnia, T. M. Rabsilber, and G. U. Auffarth, "Central and peripheral pachymetry measurements according to age using the Pentacam rotating Scheimpflug camera," Journal of Cataract and Refractive Surgery, vol. 33, no. 5, pp. 830-836, 2007.

[10] N. Q. Ali, D. V. Patel, and C. N. J. McGhee, "Biomechanical responses of healthy and keratoconic corneas measured using a noncontact scheimpflug-based tonometer," Investigative Ophthalmology and Visual Science, vol. 55, no. 6, pp. 3651-3659, 2014.

[11] Y. Hon and A. K. C. Lam, "Corneal deformation measurement using Scheimpflug noncontact tonometry," Optometry and Vision Science, vol. 90, no. 1, pp. el-e8, 2013.

[12] G. Nemeth, Z. Hassan, A. Csutak, E. Szalai, A. Berta, and L. Modis Jr., "Repeatability of ocular biomechanical data measurements with a scheimpflug-based noncontact device on normal corneas," Journal of Refractive Surgery, vol. 29, no. 8, pp. 558$563,2013$. 
[13] X. Chen, A. Stojanovic, Y. Hua et al., "Reliability of corneal dynamic scheimpflug analyser measurements in virgin and post-PRK eyes," PLoS ONE, vol. 9, no. 10, Article ID e109577, 2014.

[14] J. Katz, S. Zeger, and K.-Y. Liang, "Appropriate statistical methods to account for similarities in binary outcomes between fellow eyes," Investigative Ophthalmology and Visual Science, vol. 35, no. 5, pp. 2461-2465, 1994.

[15] J. M. Bland and D. G. Altman, "Measurement error," British Medical Journal, vol. 313, no. 7059, article 744, 1996.

[16] J. M. Bland and D. G. Altman, "Statistical methods for assessing agreement between two methods of clinical measurement," The Lancet, vol. 1, no. 8476, pp. 307-310, 1986.

[17] M. L. Salvetat, M. Zeppieri, C. Tosoni, M. Felletti, L. Grasso, and P. Brusini, "Corneal deformation parameters provided by the Corvis-ST Pachy-Tonometer in healthy subjects and glaucoma patients," Journal of Glaucoma, vol. 24, no. 8, pp. 568-574, 2015.

[18] Z. N. Al-Mohtaseb, L. Wang, and M. P. Weikert, "Repeatability and comparability of corneal thickness measurements obtained from Dual Scheimpflug Analyzer and from ultrasonic pachymetry," Graefe's Archive for Clinical and Experimental Ophthalmology, vol. 251, no. 7, pp. 1855-1860, 2013.

[19] M. J. Maldonado, A. López-Miguel, J. C. Nieto, J. Cano-Parra, B. Calvo, and J. L. Alió, "Reliability of noncontact pachymetry after laser in situ keratomileusis," Investigative Ophthalmology and Visual Science, vol. 50, no. 9, pp. 4135-4141, 2009.

[20] F. Bao, Q. Wang, S. Cheng et al., "Comparison and evaluation of central corneal thickness using 2 new noncontact specular microscopes and conventional pachymetry devices," Cornea, vol. 33, no. 6, pp. 576-581, 2014.

[21] N. Menassa, C. Kaufmann, M. Goggin, O. M. Job, L. M. Bachmann, and M. A. Thiel, "Comparison and reproducibility of corneal thickness and curvature readings obtained by the Galilei and the Orbscan II analysis systems," Journal of Cataract and Refractive Surgery, vol. 34, no. 10, pp. 1742-1747, 2008.

[22] D. Chen and A. K. C. Lam, "Intrasession and intersession repeatability of the Pentacam system on posterior corneal assessment in the normal human eye," Journal of Cataract and Refractive Surgery, vol. 33, no. 3, pp. 448-454, 2007.

[23] D. Chen and A. K. C. Lam, "Reliability and repeatability of the Pentacam on corneal curvatures," Clinical and Experimental Optometry, vol. 92, no. 2, pp. 110-118, 2009.

[24] M. R. Lattimore Jr., S. Kaupp, S. Schallhorn, and Rt. Lewis, "Orbscan pachymetry: implications of a repeated measures and diurnal variation analysis," Ophthalmology, vol. 106, no. 5, pp. 977-981, 1999.

[25] S. M. Nam, H. K. Lee, E. K. Kim, and K. Y. Seo, "Comparison of corneal thickness after the instillation of topical anesthetics: proparacaine versus oxybuprocaine," Cornea, vol. 25, no. 1, pp. 51-54, 2006.

[26] S. Amano, N. Honda, Y. Amano et al., "Comparison of central corneal thickness measurements by rotating scheimpflug camera, ultrasonic pachymetry, and scanning-slit corneal topography," Ophthalmology, vol. 113, no. 6, pp. 937-941, 2006.

[27] M. Lanza, E. Paolillo, U. A. Gironi Carnevale et al., "Central corneal thickness evaluation in healthy eyes with three different optical devices," Contact Lens and Anterior Eye, vol. 38, no. 6, pp. 409-413, 2015.

[28] W. A. Khaja, S. Grover, A. T. Kelmenson, L. R. Ferguson, K. Sambhav, and K. V. Chalam, "Comparison of central corneal thickness: ultrasound pachymetry versus slit-lamp optical coherence tomography, specular microscopy, and Orbscan," Clinical Ophthalmology, vol. 9, pp. 1065-1070, 2015.

[29] A. Smedowski, B. Weglarz, D. Tarnawska, K. Kaarniranta, and E. Wylegala, "Comparison of three intraocular pressure measurement methods including biomechanical properties of the cornea," Investigative Ophthalmology and Visual Science, vol. 55, no. 2, pp. 666-673, 2014.

[30] J. Huang, X. Ding, G. Savini et al., "Central and midperipheral corneal thickness measured with Scheimpflug imaging and optical coherence tomography," PLoS ONE, vol. 9, no. 5, Article ID e98316, 2014.

[31] M. A. Anayol, E. Güler, R. Yağci et al., "Comparison of central corneal thickness, thinnest corneal thickness, anterior chamber depth, and simulated keratometry using galilei, Pentacam, and Sirius devices," Cornea, vol. 33, no. 6, pp. 582-586, 2014.

[32] N. Maresca, F. Zeri, P. Palumbo, and A. Calossi, "Agreement and reliability in measuring central corneal thickness with a rotating Scheimpflug-Placido system and ultrasound pachymetry," Contact Lens and Anterior Eye, vol. 37, no. 6, pp. 442-446, 2014.

[33] S. Feizi, M. R. Jafarinasab, F. Karimian, H. Hasanpour, and A. Masudi, "Central and peripheral corneal thickness measurement in normal and keratoconic eyes using three corneal pachymeters," Journal of Ophthalmic \& Vision Research, vol. 9, pp. 296-304, 2014.

[34] P. De La Parra-Colín, M. Garza-León, and T. BarrientosGutierrez, "Repeatability and comparability of anterior segment biometry obtained by the Sirius and the Pentacam analyzers," International Ophthalmology, vol. 34, no. 1, pp. 27-33, 2014.

[35] J. Jorgel, J. Rosado, J. Diaz-Rey, and J. Gonzalez-Meijome, "Central corneal thickness and anterior chamber depth measurement by Sirius Scheimpflug tomography and ultrasound," Clinical Ophthalmology, vol. 7, pp. 417-422, 2013.

[36] H. A. Bayhan, S. A. Bayhan, and I. Can, "Comparison of central corneal thickness measurements with three new optical devices and a standard ultrasonic pachymeter," International Journal of Ophthalmology, vol. 7, no. 2, pp. 302-308, 2014.

[37] J. Huang, G. Savini, L. Hu et al., "Precision of a new Scheimpflug and Placido-disk analyzer in measuring corneal thickness and agreement with ultrasound pachymetry," Journal of Cataract and Refractive Surgery, vol. 39, no. 2, pp. 219-224, 2013.

[38] N. Nassiri, K. Sheibani, S. Safi et al., "Central corneal thickness in highly myopic eyes: Inter-device agreement of ultrasonic pachymetry, Pentacam and Orbscan II before and after photorefractive keratectomy," Journal of Ophthalmic \& Vision Research, vol. 9, no. 1, pp. 14-21, 2014.

[39] H. M. Al Farhan, W. M. Al Otaibi, H. M. Al Razqan, and A. A. Al Harqan, "Assessment of central corneal thickness and corneal endothelial morphology using ultrasound pachymetry, non-contact specular microscopy, and Confoscan 4 confocal microscopy," BMC Ophthalmology, vol. 13, no. 1, article 73, 2013.

[40] L.-Y. Tai, K.-W. Khaw, C.-M. Ng, and V. Subrayan, "Central corneal thickness measurements with different imaging devices and ultrasound pachymetry," Cornea, vol. 32, no. 6, pp. 766-771, 2013.

[41] S. Chen, J. Huang, D. Wen, W. Chen, D. Huang, and Q. Wang, "Measurement of central corneal thickness by highresolution Scheimpflug imaging, Fourier-domain optical coherence tomography and ultrasound pachymetry," Acta Ophthalmologica, vol. 90, no. 5, pp. 449-455, 2012.

[42] J. Aramberri, L. Araiz, A. Garcia et al., "Dual versus single Scheimpflug camera for anterior segment analysis: Precision 
and agreement," Journal of Cataract and Refractive Surgery, vol. 38, no. 11, pp. 1934-1949, 2012.

[43] J. González-Pérez, J. M. González-Méijome, M. T. Rodríguez Ares, and M. Á. Parafita, "Central corneal thickness measured with three optical devices and ultrasound pachometry," Eye \& Contact Lens, vol. 37, no. 2, pp. 66-70, 2011. 


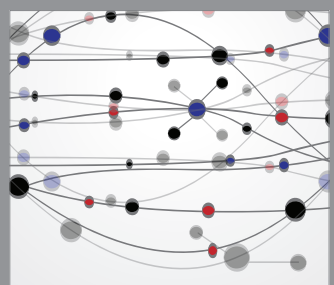

The Scientific World Journal
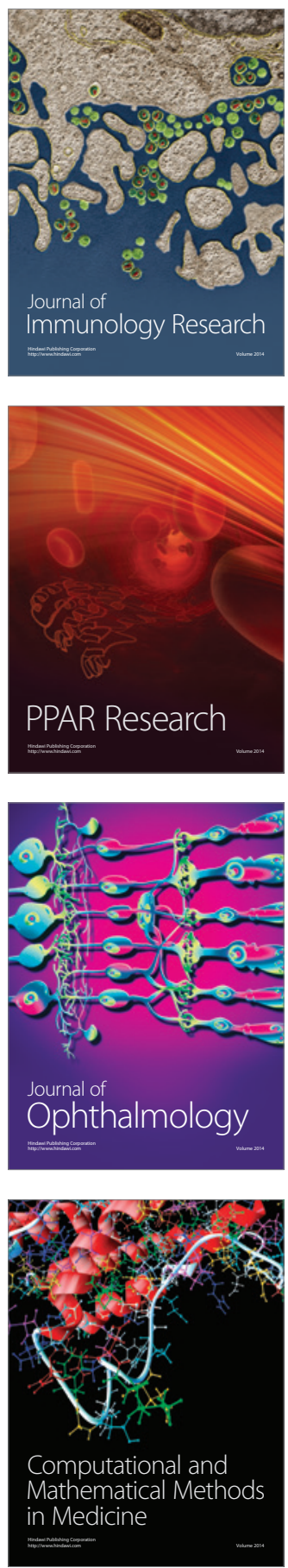

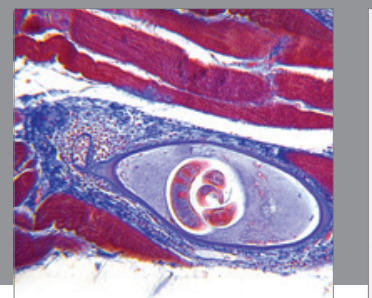

Gastroenterology

Research and Practice
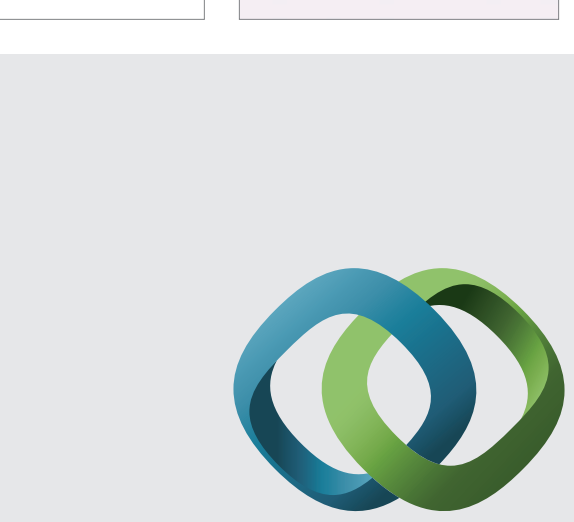

\section{Hindawi}

Submit your manuscripts at

http://www.hindawi.com
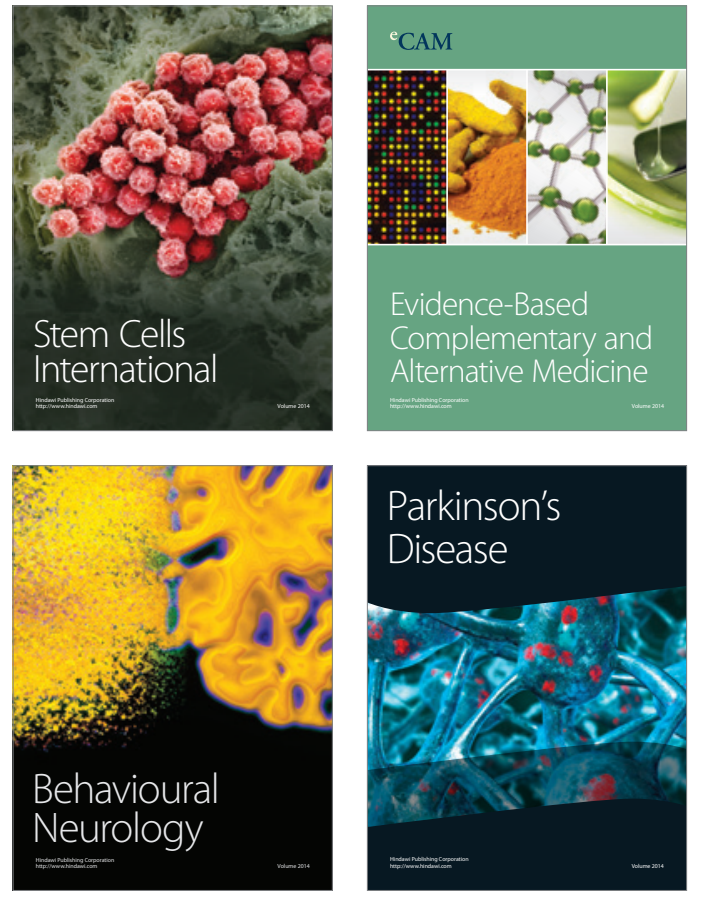
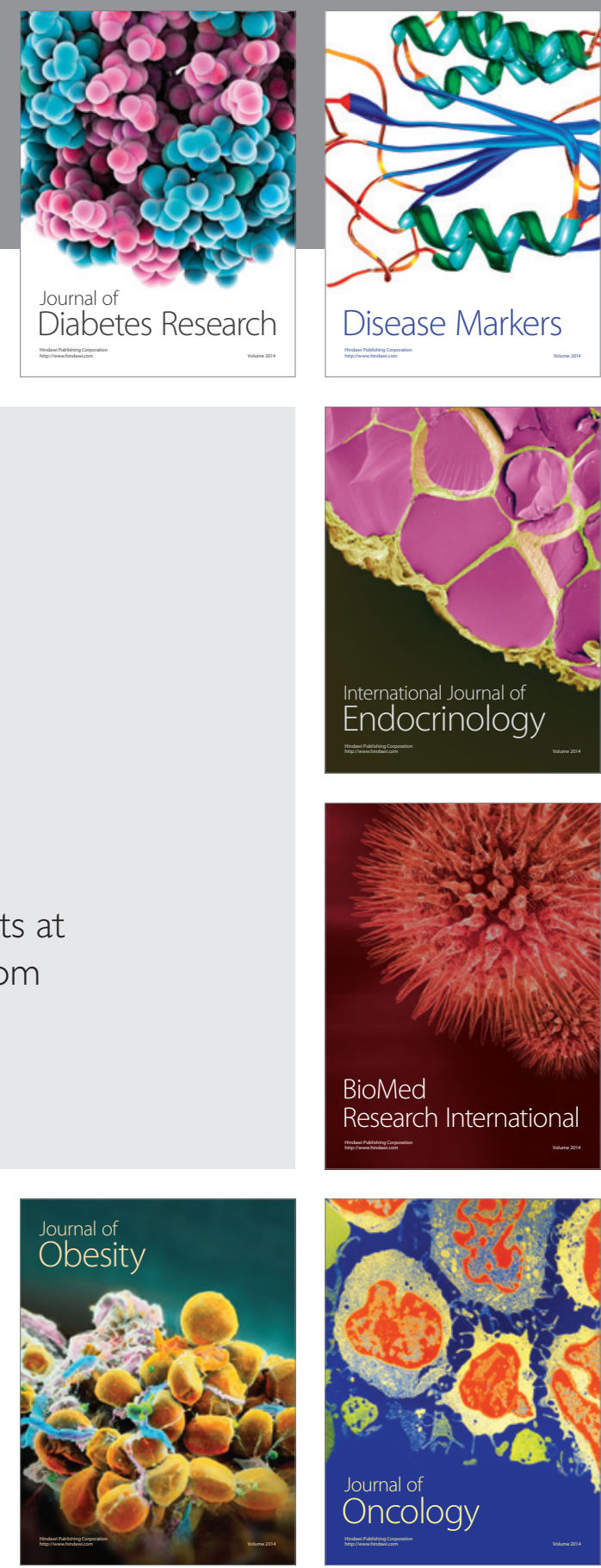

Disease Markers
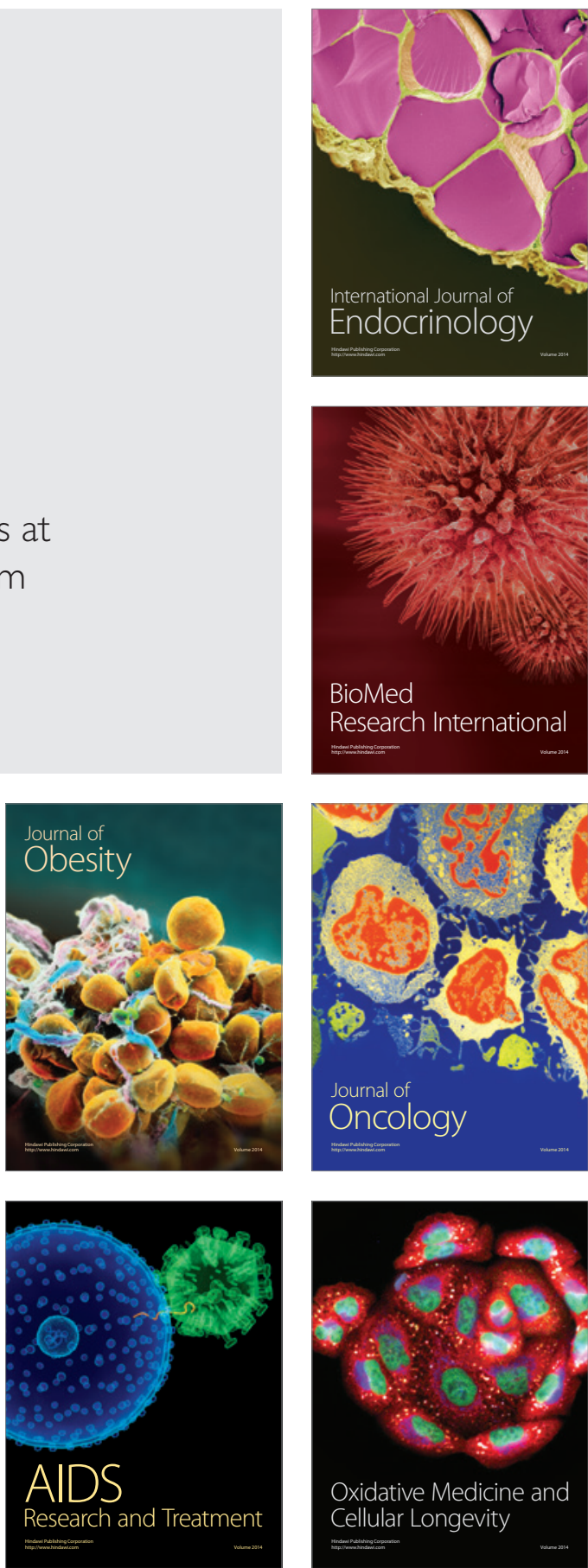